LAWRENCE LIVERMORE N A TION AL LABORATORY
Bio-Aerosol Detection Using

Mass Spectrometry: Public Health Applications

Laura D. Ludvigson

March 25, 2004 
This document was prepared as an account of work sponsored by an agency of the United States Government. Neither the United States Government nor the University of California nor any of their employees, makes any warranty, express or implied, or assumes any legal liability or responsibility for the accuracy, completeness, or usefulness of any information, apparatus, product, or process disclosed, or represents that its use would not infringe privately owned rights. Reference herein to any specific commercial product, process, or service by trade name, trademark, manufacturer, or otherwise, does not necessarily constitute or imply its endorsement, recommendation, or favoring by the United States Government or the University of California. The views and opinions of authors expressed herein do not necessarily state or reflect those of the United States Government or the University of California, and shall not be used for advertising or product endorsement purposes.

This work was performed under the auspices of the U.S. Department of Energy by University of California, Lawrence Livermore National Laboratory under Contract W-7405-Eng-48. 


\title{
Bio-aerosol Detection using Mass Spectrometry: Public Health Applications
}

\author{
Laura Ludvigson \\ Spring 2004 \\ Comprehensive Paper \\ UC Berkeley School of Public Health \\ Master's Program -Infectious Disease
}





\section{$\underline{\text { Introduction }}$}

I recently spent a summer as an intern at the Lawrence Livermore National Laboratory. I worked on a project involving the real-time, reagentless, single cell detection of aerosolized pathogens using a novel mass spectrometry approach called Bio-Aerosol Mass Spectrometry (BAMS). Based upon preliminary results showing the differentiation capabilities of BAMS, I would like to explore the development and use of this novel detection system in the context of both environmental and clinical sample pathogen detection. I would also like to explore the broader public health applications that a system such as BAMS might have in terms of infectious disease prevention and control.

In order to appreciate the potential of this instrument, I will demonstrate the need for better pathogen detection methods, and outline the instrumentation, data analysis and preliminary results that lead me toward a desire to explore this technology further. I will also discuss potential experiments for the future along with possible problems that may be encountered along the way.

\section{A Need for Better Pathogen Detection}

Many of the world's largest contributors to morbidity and mortality either cause respiratory disease or are transmitted to the human host via the respiratory tract ${ }^{4,48}$. Regardless of whether the disease is mild, like the "common cold", or more serious and potentially deadly, like pneumonic plague, the mechanism of transmission is the same. Pathogenic organisms that become aerosolized as a natural means for transmission or because of an intentional bioterrorist threat, are of major public health importance. From a public health and point of view, the best way to prevent excess disease is to stop transmission. One of the best ways to stop transmission is to have an early detection system that allows the implementation of needed control measures.

\section{Bioterrorism}

A popular focus for early detection methods is currently to protect the public from an intentional bioterrorist threat. A short list of the most common threats contains representatives of bacterial and viral species along with toxin mediated diseases. They include Anthrax, Brucella, Glanders, Pneumonic Plague, Tularemia, Q-Fever, Smallpox, Botulism, and Viral Hemorrhagic Fevers ${ }^{38}$. These possible biological warfare agents are clear examples that stress a need for early and rapid detection. Possible biowarfare agents are categorized by the CDC using 4 criteria: the ability to be easily disseminated; the ability to be spread person to person; the ability to cause significant morbidity and mortality; the ability to cause public panic and disruption ${ }^{41}$. Most of the pathogens mentioned above fall into the highest threat category, Category A. This means they are easily transmitted via aerosol or another route, they have 
very low infectious doses, and many have lethal clinical outcomes especially if undiagnosed or untreated $^{38}$. Many of these organisms also cause respiratory disease, or are transmitted via the respiratory route. The problem with current methods to detect these pathogens in clinical laboratories is the fact that many of these pathogens respond poorly and are missed using standard growth media and biochemical tests, making it difficult to have rapid diagnosis ${ }^{41}$. All of these characteristics make early detection necessary.

In the context of an aerosolized bioterrorist event, it is clear that early environmental detection of the pathogenic threat could translate into significantly fewer casualties. The resulting number of avoided casualties would be far greater than from the early detection of human disease in, for example, the release of the smallpox virus or anthrax spores. If the emergency services and public health authorities were mobilized before the threat reached a significant number of people, a preparedness and control plan could be implemented in a much shorter amount of time.

\section{Detection of Unintentional Environmental Threats}

This early detection scenario is not so different when applied to many of the unintentional pathogenic threats we face today. Infectious diseases, by definition, are spread via environmental exposure to pathogens (whether it be via person to person or environment to person); by being prepared for the detection of any environmentally spread pathogen, we can better prepare ourselves for the release of a particularly lethal one. Of particular concern in relation to a rapid detection technique leading to rapid control measures, is the causative relation of a pathogen to disease. If a known pathogen like Streptococcus pneumoniae were detected in the environment, it would be foolish to implement immediate control measures. It is a known commensal organism that frequently colonizes human hosts without causing disease. On the other hand, there are many examples of pathogens, that when detected in the environment have high potential, or can be directly linked to the cause of disease. Examples of these diseases include Tuberculosis, SARS, Influenza, Toxoplasmosis (air), Leptospirosis, Cholera, and Cryptosporidium (water and soil) ${ }^{8}$.

\section{Detection of Pathogens in Clinical Samples: focus on respiratory secretions}

The detection of pathogens from an infected (diseased or not) host is equally as important as the detection of aerosolized pathogens in the environment. In order to take necessary public health precautions such as avoiding ill persons, or avoiding shared space with those who are highly infectious, we must have early detection of the pathogen. There are many examples of respiratory pathogens to dilate on this point, but 
for the scope of this paper, I will only focus on a few that clearly demonstrate the need for better detection methods.

\section{Relevant Diseases for BAMS Technology}

The pathogens most relevant to the use of BAMS technology will be those that if detected, will have a high probability of causing significant disease that merits immediate public health control measures.

They do not include common commensal organisms or those that cause untreatable, mild and self-limiting disease (i.e. the common cold). With these criteria, I have chosen four pathogens that best represent clear benefits for early detection in the environment and/or infected host. They include two bacteria and two viruses: Legionella species, Mycobacterium tuberculosis, Influenza virus, and Respiratory Syncytial virs.

\section{Legionellosis}

Legionellosis, or Legionnaire's Disease is caused by a bacterial pathogen that is usually spread via aerosolized bacilli from contaminated water sources in a building's heating, cooling, and ventilation systems. There have been large outbreaks of Legionellosis in hotels as well as hospitals, and the morbidity/mortality associated with this infection and resulting pneumonia can be very significant (5\%$25 \%$ mortality), especially in nosocomial settings ${ }^{2}$. Clinical detection methods in hospitalized patients presenting with pneumonia are expensive (\$50-100) and not usually requested even though the diagnostic value of a positive test is significantly high. If Legionella species are detected in any respiratory secretion of someone with pneumonia, its role in disease is definite. If tests are requested, they usually include culture, serology, DFA (direct fluorescent antibody) stain, urine antigen assay, and $\mathrm{PCR}^{2}$. Culturing methods have the best detection outcomes, but are long and technically demanding. Serology is nonspecific and also takes a long time since serum antibodies in serum will appear well past infection. DFA staining is much faster, but requires more expertise and careful reagent selection. PCR is expensive, and there are no FDA-approved reagents. The urine antigen assay is rapid, easy to perform and interpret, but only detects $70 \%$ of cases. Having a more rapid and reliable detection system for both clinical and environmental samples would significantly reduce the disease burden caused by Legionella species. Having the ability to rapidly detect a sub-infectious dose of bacteria from a hospital cooling system not only could prevent large outbreaks, but it could also have large economic impacts in relation to decontamination and treatment savings. Having an early detection system in place for Legionella would also strengthen and better prepare our existing emergency response system in the event of something more fatal and/or intentional. 
Tuberculosis

Mycobacterium tuberculosis (MTB) is on of humankind's most successful pathogens, causing devastating disease throughout recorded history ${ }^{9}$. It is also an example of how a rapid detection system from both clinical samples and the environment could make a tremendous public health impact. It is highly contagious in densely populated areas, and because we do not have an early detection system for MTB in infected hosts, Tuberculosis continues to spread worldwide, causing significant morbidity and mortality. It is estimated that one third of the world's population is infected with Mycobacterium tuberculosis, with 1.6 million deaths/year and 8 million new cases diagnosed ${ }^{49}$. Mycobacterium tuberculosis is considered a very dangerous pathogen in part due to its ability to become and remain aerosolized in droplet nuclei for long periods of time. The risk of infection from exposure varies depending on environmental conditions. Casusal social contacts have a 1 in 100,000 chance of becoming infected, but that ratio is much lower if exposure occurs in schools or the workplace (1 in 3-50), nursing homes (1 in 20), bars or parties (1 in 10), dormitories ( 1 in 5$)$, or the home $(1 \text { in } 3)^{30}$.

The United States carries a relatively small amount of the total Tuberculosis disease burden, in part because of our control measures and improved living conditions. In 2002, there were about 15,000 reported cases, and over $50 \%$ of those cases occurred in foreign-born persons ${ }^{6,7}$. The main thrust to control existing and new cases of TB in the U.S. are centered around international control and screening of recent international arrivals ${ }^{5,6}$. Every year, 800,000 persons applying for long-term residence are screened either in the United States or in their home country ${ }^{5}$. The screening methods include an initial radiological screen followed by sputum smear microscopy for acid-fast bacilli (AFB) in those with positive radiological results. If someone is found to have active TB, they must be treated. In some cases, when screening is performed inside the United States, a tuberculin skin test and epidemiological contact tracing is performed ${ }^{5}$. This current screening system is inadequate for many reasons. Radiological films can be manipulated so persons coming into the country falsely use a negative X-ray as their own. Sputum smears can be falsely negative especially if the AFB load is small or the person does not have active disease. Relying on a tuberculin test can also be difficult since there is no reliable method in existence to distinguish between a positive result caused by MTB or from the widely administered BCG vaccine for $\mathrm{TB}^{5}$. For all of the above reasons, it is estimated that 7 million foreign-born persons in the U.S. are infected with MTB, up to 210,000 of which will go on to develop active $\mathrm{TB}^{5}$. To compound this problem, multi-drug resistant Tuberculosis (MDR-TB) is rapidly emerging in high TB prevalence countries, and the ability for MDR-TB to spread worldwide is very real threat. In the United States, MDR-TB among foreign-born persons increased from $31 \%$ in 1993 to $72 \%$ in $2002^{6}$. Overall, having the ability to detect and diagnose TB early would not only prevent a significant amount of morbidity and 
mortality, but it would also be very beneficial economically. Prophylaxis regimes require multiple drugs and are long (at least six months) and very expensive, especially if there is an increase in circulating MDR-TB.

Environmental detection of MTB bacilli in aerosolized droplet nuclei has also shown to be difficult, in part because the infected droplet nuclei exist in very small concentrations relative to the surrounding particulate composition $^{31}$. The only methods we have for environmental detection are epidemiological investigations of contacts of identified TB patients (aided with molecular fingerprinting techniques to differentiate similar and different strains between individuals) ${ }^{31}$. There have been some new methods developed using mircropore filters and $\mathrm{PCR}^{25}$, but for reasons explained later in this proposal, PCR may not be the best rapid detection method.

\section{$\underline{\text { Influenza }}$}

Influenza virus causes well documented worldwide epidemics every year. This is due in part to its antigenic variation strategy called genetic drift (Figure 1), in which slight genetic point mutations enhance its ability to avoid a semi-immune human population ${ }^{32}$. It is also due to the virus's very high attack rates (10\%-20\% in the general population and up to $50 \%$ in closed populations) ${ }^{8}$ Influenza causes significant morbidity and mortality, especially in the very young and old, or with immunocomprimised individuals. Every annual epidemic of influenza causes $\geq 20,000$ (range 20,000 to 40,000) deaths in the United States alone $e^{2,8}$. In the event of a completely new strain evolving from an antigenic variation strategy called antigenic shift, where large genetic differences emerge due to virus recombination (Figure 1), the entire world would be immunologicially naïve and susceptible to severe infection leading to a pandemic of influenza.

Influenza pandemics have been occurring for centuries. There have been three pandemics (1918, 1957, and 1968) during this last century, with the 1918 pandemic or "Spanish Flu" being the most severe ${ }^{29}$. The "Spanish Flu" resulted in more than 20 million deaths worldwide, unusually causing the highest mortality in young adults ${ }^{8}$. Another pandemic strain in inevitable, and a recent study looking ${ }^{29}$ at past epidemiological data, estimates that an influenza pandemic today would cause an estimated 89,000207,000 deaths, up to 734,000 hospitalizations, and up to 42 million outpatient visits in the United States alone. The U.S. economic impact, excluding disruptions in commerce and society, are estimated to total $\$ 71$ to $\$ 166$ billion dollars. Using current census bureau world population estimates, with an attack rate of $50 \%-60 \%$, and a death rate of $2 \%-4 \%$, it would translate into about 3 to 3.5 billion sick and 60 to 140 million dead in one flu season. Current detection methods include virus isolation in cell culture, and 
antigen detection using an ELISA (enzyme-linked immunosorbant assay) or DFA $\operatorname{stain}^{2}$. As is the case with any culture method, it is slow and technically demanding, and antigen detection using ELISAs or DFA has proven to be marginally sensitive and specific for influenza detection ${ }^{32}$. It would be incredibly useful to have a detection system in transportation hubs (especially international airports). Mass screening of passenger breath or respiratory secretions along with environmental air sampling on planes and/or terminals could detect anyone with infection and/or disease before they have a chance to spread it unknowingly to other hosts. A rapid detection system would be useful in a variety of settings beyond transportation hubs. It could be used in large outbreak epicenters or hospitals and health clinics for rapid infection control especially during an infection with a possible pandemic strain. A related and equally important example of how an early detection system could have been very useful is the 2003 SARS epidemic.

\section{$\underline{\text { Respiratory Syncytial Virus }}$}

Respiratory Syncytial Virus (RSV) is the leading cause of severe lower respiratory illness in infants and young children ${ }^{17}$, and is responsible for large number of the 4 million yearly infant deaths due to lower respiratory infection worldwide ${ }^{42}$. RSV also causes many forms of respiratory illness including pneumonia and acute upper and lower respiratory illness in all age groups, making it a very important human respiratory pathogen ${ }^{17,30}$. RSV is an enveloped, RNA virus belonging to the family Paramyxoviridae, and has significant antigenic variation, leading to incomplete immunity in all age groups. It is highly contagious, and is spread via large droplet aerosols or environmental exposure, thus making it the source of large outbreaks in day-care centers and hospitals ${ }^{17,30}$. Because reinfection can, and does occur, RSV causes significant morbidity and mortality, and accounts for a large economic burden to the United States ${ }^{17}$. It is estimated that around $\$ 6$ billion dollars are spent per year in the United States on lower respiratory illness in infants and children; $10 \%-20 \%$ come from RSV ${ }^{17,30}$. In the elderly, there are 14,000-62,000 hospitalizations a year from RSV, costing \$150-680 million ${ }^{17}$. Due to a lack of data on the attributable burden of RSV for respiratory illness in adults, the current morbidity and economic burden of RSV is grossly underestimated. New studies have shown that RSV may be the second leading cause (behind influenza) of influenza-like illness in adults ${ }^{17}$ in the United States. Because RSV is rarely differentiated from other viral pathogens during clinical diagnosis, the true burden of disease is underestimated and unknown at best. Diagnostic tests currently used include antigen detection using ELISA or DFA stain, or traditional viral isolation in cell culture ${ }^{2,17}$. Shortcomings of these techniques have been discussed earlier. Having a rapid, early detection system in place for RSV especially in nosocomial settings, would greatly aid in disease reduction and control. It could help target strategies for specific disease control for a number of viral pathogens that currently are not differentiated 
during diagnosis. Having a better handle on the true burden of RSV infection could lead to targeted research and development for needed vaccines and therapeutics for the many infants and children who die every year from RSV infection.

\section{Rapid Detection Techniques}

Despite a better understanding of infectious disease epidemiology and pathogenesis, rapid pathogen detection in both the external environment and in an infected (diseased) host remains a challenge. There are two important diagnostic aspects for disease prevention and control: early and rapid identification after initial infection, and pre-infection detection of harmful agents in the environment. Successful rapid detection systems that are both sensitive and specific have not yet been implemented. There are a number of promising technologies that are being used including nucleic acid-based assays and immunoassays for antigen detection. When evaluating the potential of a new test, three criteria should be met: speed, accuracy, and ease of use $\mathrm{e}^{12}$. No current diagnostic method fulfills all three criteria.

\section{PCR}

Rapid nucleic acid-based techniques like Polymerase Chain Reaction (PCR) ${ }^{15}$ have dramatically increased the time to detection over traditional culturing and biochemical methods in a number of pathogens, and in many cases, eliminated the need for culturing prior to detection, but there a number of shortfalls. (PCR) involves the long and demanding first step of extracting DNA from within cells. Traditionally, it also uses a time and labor intensive DNA/RNA quantization and labeling method using agarose gel electrophoresis ${ }^{1}$. Along with time consuming first and last steps, the sample preparation for DNA amplification is also technically demanding and very sensitive to individual laboratory conditions, making it difficult to create a standardized and reliable method across laboratories or field workers. There is also a well-documented problem with DNA contamination during successive PCR reactions, leading to false positives when amplified DNA from one sample is carried over into another ${ }^{12}$. One notable improvement to PCR based methods is the development of real-time PCR ${ }^{19-21,23}$. By incorporating the labeling and detection of nucleic acid PCR products into the amplification step, it is a much faster, more specific, quantifiable method for the detection of pathogen DNA or RNA ${ }^{1,33}$ There are also improvements with miniaturization and portability, allowing for a one-tube process, thus eliminating many possible sources of contamination ${ }^{12}$. Nevertheless, real-time PCR methods are still technically demanding, require front end sample preparation, have difficulty with the detection of multiple pathogens simultaneously, and can only provide quantitative amounts of DNA or RNA, not specific numbers of pathogens. 


\section{Microarray}

Another nucleic acid based method uses microarray technology. Microarray technology uses DNA chips that are covered with multiple (hundreds to thousands) oligonucleotides that are fluorescently labeled, and that correspond to specific segments of DNA or RNA from different pathogens. The advantage over PCR based methods, is the ability to detect multiple pathogens simultaneously ${ }^{47}$. However, current methods still require the amplification of pathogen DNA in order to produce a significant signal on the microarray chip $^{47}$. Like PCR, microarray technology can only detect pathogen DNA or RNA, and specific pathogen numbers are not known. Microarray technology is also very labor intensive and time consuming, two characteristics that for the time being, make it an inadequate method for rapid pathogen detection.

\section{Immunoassays}

There are also immuno-based methods that can detect pathogen antigens using a variety of methods. The most commonly used methods for the rapid detection of pathogens are Enzyme-Linked Immunosorbent Assays (ELISA or EIA), Immunoprecipitation, Immunofluorescence (DFA, IFA), and Flow Cytometry. All of these methods use antigen-specific antibodies labeled with various dyes (usually fluorescent) to attach to cells or cell lysates for the rapid detection of pathogens ${ }^{15}$. The use of ELISAs is widespread in clinical laboratories, but it requires a significant amount of time to set up the experiment/test. Immunofluorescent methods like DFA are attractive because they can be used directly on clinical samples like respiratory secretions, and are easily interpreted under a fluorescent microscope, but they also require a certain amount of expertise in sample preparation and reagent use. Flow cytometry is a powerful single cell analysis tool that labels cells with antigen specific antibody then counts (and collects) the cells using fluorescent methods. Although it is a fast, quantitative single cell analysis system, it takes a tremendous amount of technical skill and there is some sample preparation time needed. There have been combinations of the above immunoassays to design biosensors for the detection of multiple pathogens. There is good specificity in these systems, but the amount of pathogen needed is large (i.e. to the order of $10^{5}$ or $10^{7 /} / \mathrm{ml}$ bacteria or toxin concentrations, and $10 \mathrm{ng} / \mathrm{ml}$ of virus), and in most cases, cell lysates instead of whole cells need to be analyzed ${ }^{39}$.

\section{Mass Spectrometry as an Alternative}

Mass spectrometry may provide a better alternative to rapid pathogen detection given the shortcomings of nucleic acid-based assays and immunoassays. Mass spectrometry has been used for over 100 years, predominantly in the fields of chemistry and physics. In the most basic terms, mass spectrometry ionizes molecules and separates the ions based upon their mass-to-charge ratios. This system of analysis has proven sensitivity and specificity in terms of the mass to charge ratio of the ions generated ${ }^{11,24}$. Within 
the last 20 years, due to advances in both instrumentation and technique, mass spectrometry has exploded into the field of biological science. It is now possible to analyze large biomolecules and whole microorganisms ${ }^{11,24}$. There are many different types of a mass spectrometry instruments. Their differences include the ionizing medium, sample preparation methods, and ion collection and detection. The most common instruments used today in biological research combine MALDI (matrix-assisted laser desorption ionization) or ESI-MS (electrospray ionization mass spectrometry) with three main ion separation methods: time-of-flight (TOF), quadrupole, and selective ejection of ions from a three dimensional trapping field (ion trap or Fourier transform ion cyclotron) ${ }^{11}$. All of these methods require a significant amount of expertise and sample preparation, leading to slow identification of pathogens and their proteins. They are extremely useful for basic science research and in the developing field of proteomics, but their applications in rapid pathogen detection are limited.

\section{BAMS}

The BAMS technique I used this summer is a novel system that, in basic terms takes in bio-aerosols, including aerosolized whole pathogens, desorbs and ionizes the sized single cells, and collects and detects all (positive and negative) ions using a dual polarity time-of-flight mass spectrometer. It has many advantages over existing techniques in that it is an instantaneous, reagentless, single cell analysis system. In seconds, it can sample the air (or aerosolized liquid), pull in pathogens and generate complex spectra from each individual pathogenic cell in rapid succession. There is no sample preparation, and no reagent needed (other than electricity to power the instrument.). In a matter of seconds, BAMS has the capability to detect multiple different pathogens at very small concentrations. For this reason, it has tremendous potential as a rapid detection/diagnosis system in a variety of settings.

\section{$\underline{\text { BAMS instrumentation and sample collection }}$}

BAMS instrumentation and sample collection is relatively straightforward (Figure 2). Air and aerosolized particles are drawn into the instrument either directly from the environment, or via a diffusion drier that dries suspended particles originating from a liquid sample in a Collison nebulizer. The aerosolized particles are directed through a converging nozzle into a vacuum, at which time each particle is accelerated to its terminal velocity. This velocity is determined by its aerodynamic size, and is measured using two continuous wave laser beams that scatter light as particles pass through them. The time between the two laser's light scattering events indicates a particle's velocity and thus its size. This measurement then triggers a 7 nanosecond pulsed 266nm Nd:Yag laser that both desorbs and ionizes the particle. During the ionization process, both small molecules and fragments of larger molecules are generated. Positive and negative ion spectra are then simultaneously collected by separate time of flight 
mass spectrometers. The current mass range/limitation is about 500 Daltons in both the positive and negative direction. Mass range limitations and the implications of fragmentation will be discussed later in this paper.

\section{Mass spectral data analysis}

Mass spectral data analysis can be broken into two parts: mass spectral signature characterization and real-time detection based on a defined signature library. 1000 spectra from a known sample are taken and analyzed to generate a standard set of spectra for that known sample. Each positive and negative mass spectrum from the standard is treated as a 500-element vector corresponding to the mass/charge units. Each $n$th element vector equals the total area of all peaks +/- 0.5 Daltons of $n$. Individual spectra can be compared because similar spectra have similar vectors. Once a standard signature is developed, any unknown spectrum can be compared against the standard, using a maximum angle of difference between the unknown and standard vector as a cutoff for a similar/different assignment.

Real-time detection is accomplished by using the general signature comparison method described above, combined with a novel rules tree algorithm to further differentiate particle spectra from a complex mixture in real time. First, spectra are separated either into a microbial or non-microbial populations based on the above described method. If the non-microbial spectra match a standard already tested, it is identified; if it doesn't match, it is considered "other". For the microbial populations, a set of rules based on the presence or absence of unique peaks identified from previously acquired signatures determines to which standard the spectrum belongs. In the event that multiple standards match, the more similar negative spectrum with a corresponding positive spectrum is chosen (personal communication \{Fergenson, 2004 \#97. This allows, for instance, the differentiation of Bacillus spore species based on their predetermined presence or absence of specific peaks.

\section{Preliminary BAMS Data}

The use of BAMS for bio-aerosol detection has been demonstrated with preliminary experimental results. These results lay a promising foundation for continued research in the detection bio aerosols in both the environmental and in respiratory secretions. Experimental results to date include the ability to differentiate multiple bacterial spore species from one another, spore differentiation within complex biological and non-biological mixtures, the differentiation of non-enveloped viruses from vegetative bacterial cells, and differentiation of avirulent Mycobacterium tuberculosis (H37Ra) from Mycobacterium smegmatis. My summer project also produced some promising results exploring cell development 
differences using Bacillus atrophaeus (formerly known as Bacillus globigii and Bacillus subtilis var. niger) vegetative cells as they enter their sporulation cycle.

\section{Bacterial Spore Differentiation}

Experimental results demonstrate the ability of BAMS to differentiate between two species of Bacillus spores, Bacillus atrophaeus and Bacillus thuringenesis, from one another as well as other biological and non-biological background mixtures \{Fergenson, $2004 \# 64$ \}. Accurate differentiation of B. atrophaeus against $B$ thuringenesis occurred with $100 \%$ specificity (no false positives) and $92 \%$ sensitivity based on a rules tree algorithm using two mass spectral peaks (Figure 3). Bacillus spores were chosen primarily because of their public health importance in relation to bioterrorism, but also because they contain a large amount of dipicolinic acid (DPA $)^{43}$. This unique molecule acts a reliable marker for preliminary screening, and allows for a more refined and stratified differentiation process. B. atrophaeus is a widely accepted surrogate for the pathogenic species, Bacillus anthracis in the biodefense community, and $B$. thuringenesis is a non-pathogenic species (for humans) commercially used as a pesticide. Both species, along with B. cereus, a common opportunist and cause of foodborne illness ${ }^{8}$, are very similar genetically, and some researchers suggest that they should taxonomically be considered more related ${ }^{18}$. They differ mainly with respect to plasmid mediated toxin producing virulence factors of B. anthracis ${ }^{36,37}$. Given such similarity between different Bacillus species, the ability to reproducibly differentiate B. atrophaeus from $B$. thuringenesis with $100 \%$ specificity gives strength to the BAMS technique as a useful tool for the differentiation of similar pathogens.

In addition to differentiation of pure spore samples, BAMS also accomplished Bacillus spore differentiation and detection within complex mixtures. The mixtures included fungal and bacterial (Clostridia sp.) spores to further demonstrate the technique's ability to detect different bio-aerosols. The mixtures also consisted of different growth media, suspicious white powders like Equal ${ }^{\mathrm{TM}}$, gelatin, and Gold Bond Medicated Powder ${ }^{\mathrm{TM}}$, and other common environmental aerosols including cigarette and wood smoke, Urban Particulate matter (standards), Diesel Particulate Matter, and ambient air particles from outside the laboratory. Using the BAMS analysis software previously described to generate unique mass spectral signatures for some of the complex mixture components along with the Bacillus spore signatures, a real-time detection and identification system for the spores was able to correctly differentiate B. atrophaeus from B. thuringenesisand the other background particles ( Figure 4). This set of experiments demonstrates two main strengths of the BAMS system: the ability to reproducibly generate unique mass spectral signatures for multiple Bacillus spore species, and to successfully identify them within a complex mixture of biological and non-biological particles. These experiments have 
implications for the ability of BAMS to differentiate other pathogens at the species level. It also provides strong evidence that pathogen detection using BAMS can be successful within a complex mixture.

Having the detection ability within a complex mixture is one of the most important characteristics of this kind of instrument, both in the context of the environment and host secretions (i.e. clinical samples). The early success of this differentiation capability provides a strong argument for the continuation of pathogen detection research.

\section{Mycobacterial Differentiation}

Preliminary experiments have also been performed to differentiate a Mycobacterium tuberculosis strain (H37Ra) from a non-pathogenic (saprophytic) species of Mycobacteria, and from other bacterial species $^{34,35}$. Pure cultures of H37Ra, M. smegmatis, B. atrophaeus, and E. coli were each analyzed by BAMS to generate standard spectra. The spectra were then analyzed via a rules-tree algorithm to identify peaks unique to H37Ra. One unique peak, $-421 \mathrm{~m} / \mathrm{z}$, was identified for H37Ra. Based on this peak, H37Ra was positively identified from the other bacterial species $40 \%$ of the time with no false positives (Figure 5). Following identification of this unique peak, H37Ra was aerosolized into a bio-aerosol chamber to determine how many cells could be detected per liter of air. Andersen impactor and filter samples were collected in order to determine the total cell counts via standard plate counts and quantitative PCR respectively. It was determined that with the aerosolization of 1000 cells $\left(10^{3}\right), 25$ could be detected within 12 minutes, one third of which were detected in the first minute. Given the small scope and timeframe of the experiment, these preliminary results are very promising for continued Mycobacterium tuberculosis work. Given the magnitude of TB disease morbidity worldwide, any experiment that has a positive result in terms of TB detection and species level differentiation provides a strong argument for continued research. Given the past success of Bacillus spore experiments coupled with these preliminary TB results gives strength to the ability of BAMS to detect differences between multiple pathogens at the species level.

\section{Differentiation of Viruses from Bacteria}

Other preliminary results involve the differentiation of viruses from bacteria. In some abbreviated experiments, BAMS can differentiate between a non-enveloped RNA virus (bacteriophage), MW2, and a vegetative bacterial cell. The virus does not have sodiated phosphate peaks as seen with vegetative Bacillus atrophaeus cells (personal communication ${ }^{34}$ ) (Figure 6). More work is needed in this area, and it will be a focus of future experiments. Having the ability to generally differentiate bacteria from viruses has big implications in a clinical setting. A well documented problem in the treatment of respiratory illness is the over prescription of antibiotics for non-bacterial infections ${ }^{16}$. With the ability to 
immediately differentiate a bacterial versus viral cause of disease, a more direct and targeted control effort can be implemented especially in terms of treatment options (antibiotics, antivirals, supportive). If BAMS can help improve the proper treatment of disease, positive outcomes would span beyond just treatment. A more effective, targeted treatment plan would decrease the cost of failed treatment, and dampen the emergence of drug resistant bacteria caused by inappropriate antibiotic treatment of viral illness.

\section{Cell Development Experiments}

My summer project used BAMS to study cell development. I used a model of bacterial sporulation to demonstrate mass spectral differences during different stages of sporulation . Preliminary work demonstrating the ability to differentiate 4 stages of bacterial spore development has been performed, and is in the process of more in-depth analysis. Using a set of Bacillus atrophaeus sporulation mutants, I can differentiate the following four stages in development: vegetative cell, early sporulation, mid/late sporulation, and complete spore (Figure 7). This work has promising implications for the future of BAMS in cell development. There are applications that span infectious and chronic disease. If BAMS can differentiate cells in a unique developmental state (i.e. cancerous human cell), it may be a very powerful system for the pre-symptomatic detection of cancer. The detection of Anthrax in both the environment and host is an example of the possible benefits in relation to infectious disease. If BAMS can detect what stage a B. anthracis cell is in (i.e. spore, sporulation, or germination), the information can be used to better direct a public health response. If germinating cells were detected from a clinical sample, it would signify recent anthrax spore infection and allow better patient treatment, and a faster more directed response to find Anthrax spores in the environment.

\section{The Problems and the Improvements}

Although the BAMS technique has demonstrated promise for use in pathogen detection, it is a novel system, and there are possible problems that must be addressed and dealt with if this technique is to emerge as useful.

\section{Mass Range and Fragmentation}

Currently, the biggest challenge for BAMS is the limited mass range the instrument can detect. The current instrument was adapted from an instrument originally set up for environmental analysis ${ }^{14}$, and having the ability to detect high mass proteins and other complex molecules was not necessary. Currently, the mass spectrometer can detect ions up to 1200 Daltons (Da) (well below proteins and most peptide fragments), and in most circumstances, reproducible detection has only been achieved in the $+/$ - 
500 Da range. Peaks that have been identified have all been metabolites or small molecule fragments from the ionizing process. Metabolite detection may be misleading due to fluctuations during changing microbial growth conditions, harvesting time, and environmental exposure. Many bacterial and viral pathogens may also share many common components, and identifying differences may be challenging. Alternatively, natural biologic variability within the same species given the current mass range may distort or overwhelm real species-to-species differences. Having said that, even with these challenges, the previous BAMS experiments show that reproducible differences (though few) still exist at such a small mass range. Molecular fragmentation does not pose such a challenging problem. As was demonstrated with the BAMS bacterial spore work, if there are unique biomolecules like dipicolinic acid (DPA) in bacterial spores, unique fragments can be generated. This may also be the case for mycobacterial species given their unique cell wall components.

In order to improve the mass range of the BAMS instrument, it might be useful to explore using a matrix based and/or dual laser system for the desorption and ionization process. In order to keep the instantaneous feature of the instrument, using either coated matrix particles or a second matrix beam along side the particle beam would be the preferable methods. Having two lasers, one to desorb and the other to ionize, would also allow the generation of higher mass ions with less fragmentation. Using a desorbing laser that is less powerful (i.e. IR laser) would keep ions larger. Using an ionizing laser with more power would insure a better yield of large ions once a particle has been desorbed. Creating larger ions would allow BAMS to produce protein and protein fragment peaks that may act as more reproducible and identifiable markers.

\section{Instrument Variability}

Another possible problem is the instrument variability. The generation of a mass spectral signature depends on two things: desorption/ionization laser wavelength and fluence (laser power). Both the wavelength and fluence is currently tailored to maximize bacterial spore spectra. The $266 \mathrm{~nm}$ wavelength is absorbed well by DPA in the spore, but using the same wavelength to look at other pathogens may not optimize their spectra. The fluence, which can be changed easily on the instrument, was also tailored to maximizing the DPA signal of spores. Although it can be easily changed, if the main goal is to detect a variety of different pathogens at the same time, there will need to be a set fluence that works well for a variety of organisms. From an instrumentation standpoint, even with standard laser fluence, heterogeneity still exists. The laser beam is not flat and the spectra generated from the laser pulse will depend on where the aerosolized particle comes in contact with the laser beam. Both wavelength and fluence variability may mask true biological differences that may be present. These issues are currently 
being investigated and improvements are being tested by various members of the BAMS instrumentation and laser teams ${ }^{44,46}$.

\section{Sensitivity and Specificity}

The sensitivity and specificity of the BAMS system may also pose a challenge. Inherently, a single cell analysis system is $100 \%$ sensitive, but there are variables that can decrease this sensitivity. One of these variables is the goal to maximize specificity. It is especially important for a detection and diagnostic system to have high specificity to prevent false positives. In an environmental detection application for the intentional release of an aerosolized pathogen, minimizing false positives (alarms) is important for effective public safety and response. In a clinical diagnostic setting, specificity is also important in relation to patient treatment and infection control options. In order to avoid false positives, the BAMS analysis software sets strict matching criteria (small vector angle and unique peak area differences between spectra) for an unknown and standard spectrum. In order to keep specificity high, the sensitivity of the system may be compromised due cells with unassigned spectra being missed.

Another variable effecting sensitivity involves instrumentation. A bio-aerosol detection system's limit of detection is frequently measured in terms of agent-containing particles per liter of air (ACPLA) ${ }^{13}$. The BAMS instrument currently processes 2 particles/second in urban background conditions, and has an intake of $1 \mathrm{~L}$ of air/minute. Based upon both this data processing speed and rate of air flow, BAMS can detect concentrations of $10^{4}$ ACPLA in 1 minute. Instrument improvements in multiple areas are currently underway, and will result in an instrument that is 4-5 orders of magnitude more sensitive (personal communication ${ }^{10}$ ). These improvements include a better air intake system that involves a preconcentration step using a virtual impactor, better particle tracking using multiple scattering lasers, better data handling by incorporating a preprocessing step before data storage, and a prescreening system for biological particles using fluorescence.

\section{Differentiation Complex Clinical Samples}

Aside from the possible problems arising from instrumentation shortfalls, we may also be unable to differentiate complex biological mixtures from pathogens. Although there has been success with pathogen detection within an environmental air sample, it remains to be determined if the same results will hold true for respiratory secretions. Saliva and sputum are both very complex, and interactions between their molecules, cells and infecting pathogens may be too complex to differentiate using the current BAMS instrument. Pathogens may clump with, or be surrounded by these cells or molecules, and this may generate a mass spectral signature that is different from the pathogen standard previously 
generated. An alternative to unmodified sample analysis could be to add a simple sample preparation step in order to isolate possible pathogens from the respiratory mixture. Any sample preparation step would have to be rapid and easy to perform in order to maintain the ability of BAMS to be the most rapid system available.

\section{Pushing Forward}

Although there are formidable challenges facing the success of BAMS for rapid pathogen detection, we should not dismiss the potential for its success. The BAMS instrument, with its capabilities and limitations will provide a framework and define the experimental parameters that should be set at each successive research step. As the instrument capabilities improve, more advanced research can occur.

\section{Future Experiments}

Given the success of preliminary experiments using a variety of pathogen detection models, I think more research is warranted. Keeping in mind both the current limitations and future improvements to the BAMS instrument, two approaches to pathogen detection will need to be taken. The first is a shotgun approach to detect spectral differences between pathogens. This would involve thousands of spectra from a pure pathogen sample being taken and analyzed to determine differences between unidentified ion peaks. The second approach to be used on its own, or in conjunction with the initial shotgun approach, would be to target certain molecules that may be unique to the pathogen and try to identify the ion that is generated from that molecule or molecule fragment. Given the current limitations of the BAMS system, I think both approaches will be necessary. The mass range is limited to too small ions, and the unique proteins for many pathogens are way beyond the size limitation to be detected. At this point the only way to detect large unique proteins is to detect smaller fragments generated from the ionizing process. Although this approach has had some success with the detection of DPA fragments in Bacillus spores, the ideal instrument would be able to detect non-fragmented molecular ions. When the BAMS mass range is improved, ion identification will be easier. The initial shotgun approach will still need to be used, but once ions are generated and unique peaks are found, they can be more easily identified using current MALDI and 2-D electrophoretic methods ${ }^{26-28}$. Once sequences mass of peaks are known, they can be identified using electronic genome and protein searchable databases provided by Swissprot and the Sanger Institute.

\section{Stage I: Pathogen Selection.}

Future experiments should start with a defined set of pathogens beyond the pathogens tested previously for in-depth signature development and differentiation capabilities. At this stage in instrument 
development, pathogens with unique biochemical properties will be the best choice in order to maximize the ability to detect mass spectral differences like was done for the detection of DPA in Bacillus spores. Selection should also be based in part on public health importance and benefit as it relates to the use of the BAMS technique. As eluded to previously in this paper, a virus like rhinovirus, that commonly causes the common cold, will not be pursued. Even if BAMS could detect this virus, the disease is self limited and there are no good therapeutic options once diagnosed ${ }^{8}$. Pathogens like Mycobacterium tuberculosis or Yersinia pestis should be selected based on clear benefits for early detection in terms of treatment, and transmission control. Selection should also be based on the site of transmission and primary disease manifestation (i.e. the respiratory tract), and the ability to be aerosolized. Pathogens should be composed of representative bacteria (Gram-positive and Gram-negative, mycobacterial species, mycoplasma), and viruses (RNA and DNA). Most of the pathogens I mention below can all be analyzed under standard Biosafety Level 3 conditions, of which we will be given access to on-site, or at a yet-to-be determined location. Lawrence Livermore National Laboratory has a Biosafety Level 2 facility in which many of these organisms can be grown, but not aerosolized.

The pathogens that could be included are:

Bacterial:

- Gram-positive: Bacillus anthracis: possible bioterrorist agent; continued work on identification of unique peaks.

Legionella pneumophila: public health importance and model system for environmental detection.

- Gram-negative: All of these organisms are potential bioterrorist agents with major public health implications in the event of their release.

Yersinia pestis, Burkholderia mallei, Francisella tularensis, Coxiella burnetii

- Mycoplasma pneumoniae: common cause of pneumonia in young adults, has unique biological characteristics distinct from its Gram-positive ancestors (no cell wall, dependent on sterols for adequate growth)

- Mycobacterial: Mycobacterium tuberculosis, Mycobacterium bovis, Mycobacterium avium complex

(MAC): These species cause the most frequent disease of all the mycobacteria.

Differentiation between them and from other saprophytic species like M. smegmatis will be necessary. As a group, the mycobacteria are unique in their glycolipid cell wall composition. 
Viral

The species level differentiation of viruses may prove to be more difficult than for bacteria. The differences BAMS has obtained in bacteria to date are believed to be mostly metabolite differences, and the only identified peak difference is the presence or absence of DPA in Bacillus spores versus vegetative bacteria. Give the nature of the differences observed in bacteria, I would expect viruses to pose a bigger problem. Viruses are dependant on a host to perform many of the biologic processes required for survival. Because viruses use the host cell machinery to perform these tasks, they have a relatively simple biochemical makeup composed of DNA or RNA, and proteins that make up their capsid. Enveloped viruses have added proteins and lipids, but compared to bacteria, are still very simple. Given the simplicity of viruses, I would expect to see fragments of unique proteins from the viral capsid or envelope, and maybe peaks that correspond with either DNA or RNA. We may also be able to detect specific viral types of DNA called CpG DNA??? If the BAMS capabilities can incorporate the analysis of host cells, we may be able to detect foreign viral proteins within an infected host cell, as well as changes in the immunological molecule production of host cells. Some of the most important viral pathogens that can be found in respiratory secretions or are important as aerosolized bioterrorist agents are:

- RNA virus: Influenza subtype A, coronavirus (SARS), respiratory syncytial virus (RSV), HIV*, Rabies, Viral Hemorrhagic Fever (VHF) viruses - Ebola, Marburg (Filoviridae), Lassa fever (Arenaviridae), Hantaviruses (Bunyaviridae), Dengue, Yellow Fever (Flaviviridae)

- DNA virus: Orthopoxvirus (Vaccinia virus, Monkeypox), Herpesviridae family* (Cytomegalovirus, Human herpesvirus-6,7,8, Epstein-Barr virus)

*these viruses have been shown to be present in the saliva in some hosts ${ }^{45}$.

\section{Stage II. Signature Development}

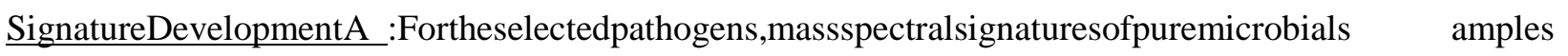
will be generated and compared to each other (and if applicable, to other non-pathogenic species of the same genus) under a variety of conditions. This set of conditions will include but not be limited to, wet (suspended in distilled, de-ionized water) and lyophilized samples grown in a variety of standard and radio-labeled growth media. Temperature conditions will also be varied. For bacterial species, cells will be harvested at their mid-log growth phase and immediately run through the BAMS instrument. Viruses will be grown according to established growth protocols and run through BAMS. The mass spectral signatures obtained will be analyzed and peaks will be identified (as many as possible) using a variety of methods including radio labeling. 
Signature Development B: Once signature standards have been developed and analyzed on a select group of pathogens, we will determine the level of differentiation we can achieve based on unique mass spectral peaks. Based on the differentiation results, we will determine to what extent BAMS can be used for detection and diagnosis. The first level of differentiation we must achieve is to differentiate between bacterial and viral samples. This has already been demonstrated with non-enveloped viruses and bacterial cells, but more work including the use of different viruses (enveloped and non-enveloped) will need to be done. The next step will be to differentiate between pathogenic versus non-pathogenic species of the same genus. This has already been done for different Bacillus spore species, but other representative pathogens must also be able to show this level of differentiation. In the event that there is sufficient species level differentiation, we will attempt to do some strain specific differentiation with a select group of pathogenic species (Mycobacterium tuberculosis outbreak strains, MDR-TB strains, influenza strains, RSV strains).

\section{Stage III: Complex Mixtures}

Once clear differences between pathogenic spectra are established, we can advance to the next stage: detection within a complex mixture. There are two important complex mixtures that would need to be tested: environmental and biological (host).

A significant amount of environmental data has already been taken from past and current BAMS research experiments ${ }^{13}$ involving Bacillus species, but these same experiments should be performed for a variety of other pathogens to confirm that the same level of detection within a complex air sample can be achieved. The same environmental complex mixtures that were used previously could be used again. One of the main focuses of this section should be the detection of viral pathogens in complex air samples because there has been no previous work performed, and it would seem likely that virus may interact with particulate matter in a different manner than bacteria.

The second set of complex mixture experiments should involve biological complex mixtures from host secretions. The focus should be on respiratory secretions including sputum, saliva, and exhaled breath. Since pathogen signatures will already be available, a standard set of signatures for uninfected "clean" biological samples should be created. These samples, for simplicity's sake can be at first, artificial mixtures of saliva and sputum. There are many commercially available artificial saliva mixtures ${ }^{40}$ that contain the principle families of active and known molecules like mucins, amylase, and other glycoproteins. In addition, other possible molecules and cells that could be found in saliva and sputum 
include growth factors, cytokines, immunoglobulins, blood cells, respiratory epithelial cells and their products $^{22}$. There is an instrument-determined particle size limitation based on the size of the BAMS nozzle that will only allow cells and particles $<5 \mu \mathrm{m}$. This limitation will initially exclude the analysis of some large cells in the mixture, but the cell products may be analyzed if there is cell lysis. In the future, different nozzle sizes can be use to incorporate larger cells. In order to aid in the artificial saliva signature development, pure samples of each mixture component may need to be run through BAMS. Once clean signatures are developed, the next step would be to combine the artificial respiratory samples with pathogen samples to determine the achievable level of detection (sensitivity and specificity).

Continued research could then test levels of detection within a more natural host background that includes common commensal bacterial flora of the mouth and upper airways. For the success of BAMS in clinical pathogen detection, it will be crucial to have the capability to differentiate pathogenic bacterial species from non-pathogenic bacterial and viral species that continually colonize the upper respiratory tract without causing disease in the host.

With the successful completion of experiments using artificial secretions, more advanced research could be started, and could include the analysis of real clinical samples from infected and control subjects. The experimental framework would follow the same protocol as was used for the artificial mixture. Future experiments could also include the analysis of other clinical samples including blood, urine, and cerebral spinal fluid. There are many steps and goals that need to be achieved before this kind research is done, but with a well organized plan, successful instrument improvements, and a realistic time-line, I think these types of experiments will be possible.

\section{Conclusion}

Public health applications using BAMS for pathogen detection are numerous and on multiple levels. They fit into multiple rubrics of disease control and prevention including early detection, treatment, surveillance, and research. After the careful evaluation of the BAMS technique, I think its applications to public health have different timelines depending on the question it is being used to answer. Due to current limitations, I think that the first real use for BAMS will be with bacterial pathogen detection. There may be some general differentiation capabilities between viruses and bacteria, but I think specieslevel identification of viruses is further down the road after improvements with instrumentation. The detection of viruses (and bacteria) will also be greatly aided by the ability of BAMS to analyze host cells and their contents. This will open doors to the detection of immune responses not only to a wide range of infectious diseases, but also to chronic conditions such as cancer. 
For a complete and reliable system for infectious disease prevention and control, three criteria must be met. There must be early detection, the effective dissemination of information from that detection, and a strong public health infrastructure to act on the information it receives. As I demonstrated earlier in this paper, having a surveillance and/or detection system using BAMS for diseases such as Legionellosis, Tuberculosis, Influenza, and Respiratory Syncytial Virus has the possibility to significantly reduce the morbidity and mortality associated with these diseases.

The detection of these pathogens in either the outdoor environment or within closed settings such as hospitals, international travel hubs, prisons, and large hotels or business buildings is a key to the prevention of large outbreaks. Using a system like BAMS has benefits over other environmental samplers. It can be used in both outdoor and indoor settings, as well as detection within clinical samples. This multi-faceted detection approach can drastically increase the chance pathogen detection from a natural or intentional source.

The detection of pathogens from clinical samples not only has implications for disease prevention, but it also has more far reaching implications for treatment options. If BAMS can detect multiple pathogens of both viral and bacterial origin, there can be drastic improvements to the way common respiratory infections are treated. Even if the ability to differentiate is limited to a viral or bacterial prognosis, the decision to prescribe antibiotics can be based on more solid diagnostic information. I think that realistically, this differentiation will be one of the greatest clinical benefits of BAMS, in terms of both health and cost benefits.

Again, the use of BAMS for the early detection of pathogens is only the first step in an adequate attempt for disease control and prevention. The public health response to a possible disease threat is a complicated puzzle with many pieces that need to fit together in order to be complete. If some of the pieces or missing, having an early detection system will not be a great benefit to the public's health in the long run. There needs to be and intertwined public health system that incorporates infectious disease research, early detection, information management, a clear organizational structure, and the funds and ability to take action. This system needs coordinated efforts in the political as well as scientific world.

Because of the need for more than just a rapid detection system for the effective use of BAMS, I think the best first step is its introduction into the developed world. In order to convince the public and maximize the chance for successful pathogen detection leading to public health prevention and control, BAMS will 
need to start out in countries and settings that have existing public health infrastructure and funding to act on the information provided by BAMS. Whether that action is the deployment of rapid control measures after a bioterrorist threat or the initiation of complete and effective treatment once a pathogen has been associated with a particular disease in a host, having the ability to act is crucial to success. Unfortunately, many developing countries around the world do not have the wealth or infrastructure to take full advantage of the benefits from this technology. They do, however, have the highest burdens of disease morbidity and mortality, especially for diseases like Tuberculosis and Respiratory Syncytial Virus. In the event of a bioterrorist attack, these same countries would be in disarray trying to deal with the sick using inadequate facilities and supplies. The dire circumstances for developing countries in the event of a natural or intentional disease outbreak gives technologies like BAMS even more reason to succeed. If BAMS is to be a truly successful bio-aerosol detector, this technology must be usable to those who will benefit the most from early detection and diagnosis, not just the wealthy. There is always a possibility that this technology will only be applicable to a select few due to cost and ease of use, and that is why we must take any opportunity that exists to maximize the chances for the success of BAMS in rapid pathogen detection. With accomplishment in the developed world, we can push forward to make it a successful detection method worldwide. 


\section{$\underline{\text { References }}$}

1. ABI Applied Biosystems. (2004). Real-time PCR vs. Traditional Block PCR. Retrieved March, 2004, from http://www.wzw.tum.de/gene-quantification/abi-rtpcr-pcr.pdf

2. Bartlett, J. G., Breiman, R. F., Mandell, L. A., \& File, T. M., Jr. (1998). Communityacquired pneumonia in adults: guidelines for management. The Infectious Diseases Society of America. Clin Infect Dis, 26(4), 811-838.

3. Bio Aerosol Mass Spectrometry Laboratory Group. (2002). BAMS Instrument Schematic.

4. Brock, T. (Ed.). (1997). Biology of Microorganisms(8th ed.). Upper Saddle River, NJ: Prentice Hall.

5. CDC. (1998). Recommendations for Prevention and Control of Tuberculosis Among Foreign-Born Persons. Morbidity and Mortality Weekly Report, 47(No. RR-16).

6. CDC. (2004). Reported Tuberculosis in the United States, 2002 - Executive Summary. Retrieved 3/1/2004, from http://www.cdc.gov/nchstp/tb/surv/surv2002/default.htm

7. CDC. (2004). Tuberculosis Cases in Foreign-born Persons by Country of Origin: States, 2002. Retrieved 3/1/04, from http://www.cdc.gov/nchstp/tb/surv/surv2002/default.htm

8. Chin, J., \& American Public Health Association. (2000). Control of communicable diseases manual : an official report of the American Public Health Association (17th ed.). Washington, DC: American Public Health Association.

9. Clark-Curtiss, J. E., \& Haydel, S. E. (2003). Molecular genetics of Mycobacterium tuberculosis pathogenesis. Annu Rev Microbiol, 57517- 549.

10. Coffee, K. R. (2004). BAMS Instrument Design: current and future. Livermore, CA.

11. Dalluge, J. J. (2000). Mass spectrometry for direct determination of proteins in cells: applications in biotechnology and microbiology. Fresenius J Anal Chem, 366(6-7), 701711.

12. Enserink, M. (2001). Anthrax. Biodefense hampered by inadequate tests. Science, 294(5545), 1266-1267.

13. Fergenson, D. P., Pitesky, M. E., Tobias, H. J., Steele, P. T., Czerwieniec, G. A., Russell, S. C., et al. (2004). Reagentless detection and classification of individual bioaerosol particles in seconds. Anal Chem, 76(2), 373-378.

14. Gard, E. E., Kleeman, M. J., Gross, D. S., Hughes, L. S., Allen, J. O., Morrical, B. D., et al. (1998). Direct observation of heterogeneous chemistry in the atmosphere. Science, 279(5354), 1184-1187.

15. Goldsby, R., Kindt, T., \& Osborne, B. (2000). Kuby Immunology (4th ed.). New York: W. H. Freeman and Company.

16. Gonzales, R., Malone, D. C., Maselli, J. H., \& Sande, M. A. (2001). Excessive antibiotic use for acute respiratory infections in the United States. Clin Infect Dis, 33(6), 757-762.

17. Hashem, M., \& Hall, C. B. (2003). Respiratory syncytial virus in healthy adults: the cost of a cold. $J$ Clin Virol, 27(1), 14-21.

18. Helgason, E., Okstad, O. A., Caugant, D. A., Johansen, H. A., Fouet, A., Mock, M., et al. (2000). Bacillus anthracis, Bacillus cereus, and Bacillus thuringiensis--one species on the basis of genetic evidence. Appl Environ Microbiol, 66(6), 2627-2630.

19. Higuchi, R., Dollinger, G., Walsh, P. S., \& Griffith, R. (1992). Simultaneous amplification and detection of specific DNA sequences. Biotechnology (N Y), 10(4), 413-417.

20. Higuchi, R., Fockler, C., Dollinger, G., \& Watson, R. (1993). Kinetic PCR analysis: realtime monitoring of DNA amplification reactions. Biotechnology (N Y), 11(9), 1026-1030. 
21. Holland, P. M., Abramson, R. D., Watson, R., \& Gelfand, D. H. (1991). Detection of specific polymerase chain reaction product by utilizing the 5'----3' exonuclease activity of Thermus aquaticus DNA polymerase. Proc Natl Acad Sci U S A, 88(16), 7276-7280.

22. Lawrence, H. P. (2002). Salivary markers of systemic disease: noninvasive diagnosis of disease and monitoring of general health. J Can Dent Assoc, 68(3), 170-174.

23. Lee, L. G., Connell, C. R., \& Bloch, W. (1993). Allelic discrimination by nick-translation PCR with fluorogenic probes. Nucleic Acids Res, 21(16), 3761-3766.

24. Mann, M., Hendrickson, R. C., \& Pandey, A. (2001). Analysis of proteins and proteomes by mass spectrometry. Annu Rev Biochem, 70, 437-473.

25. Mastorides, S. M., Oehler, R. L., Greene, J. N., Sinnott, J. T. t., Kranik, M., \& Sandin, R. L. (1999). The detection of airborne Mycobacterium tuberculosis using micropore membrane air sampling and polymerase chain reaction. Chest, 115(1), 19-25.

26. Mattow, J., Jungblut, P. R., Muller, E. C., \& Kaufmann, S. H. (2001). Identification of acidic, low molecular mass proteins of Mycobacterium tuberculosis strain H37Rv by matrix-assisted laser desorption/ionization and electrospray ionization mass spectrometry. Proteomics, 1(4), 494-507.

27. Mattow, J., Jungblut, P. R., Schaible, U. E., Mollenkopf, H. J., Lamer, S., Zimny-Arndt, U., et al. (2001). Identification of proteins from Mycobacterium tuberculosis missing in attenuated Mycobacterium bovis BCG strains. Electrophoresis, 22(14), 2936-2946.

28. Mattow, J., Schaible, U. E., Schmidt, F., Hagens, K., Siejak, F., Brestrich, G., et al. (2003). Comparative proteome analysis of culture supernatant proteins from virulent Mycobacterium tuberculosis H37Rv and attenuated M. bovis BCG Copenhagen. Electrophoresis, 24(19-20), 3405-3420.

29. Meltzer, M. I., Cox, N. J., \& Fukuda, K. (1999). The economic impact of pandemic influenza in the United States: priorities for intervention. Emerg Infect Dis, 5(5), 659671.

30. Musher, D. M. (2003). How contagious are common respiratory tract infections? N Engl J Med, 348(13), 1256-1266.

31. Nardell, E. A. (2003). Environmental infection control of tuberculosis. Semin Respir Infect, 18(4), 307-319.

32. Nicholson, K. G., Wood, J. M., \& Zambon, M. (2003). Influenza. Lancet, 362(9397), 1733-1745.

33. PE Biosystems. (2004). DNA/RNA Real-Time Quantitative PCR. Retrieved March, 2004, from http://www.wzw.tum.de/gene quantification/pe-realtimeoverview-1.pdf

34. Pitesky, M. E. (2004). BAMS Mycobaterium experiments: results to date. Livermore, CA.

35. Pitesky, M. E., Fergenson, D. P., Tobias, H. J., Shaffer, M., Horn, J. M., Frank, M., et al. (2004, March 7-10). Detection of aerosolized Mycobacterium tuberculosis (H37Ra) in seconds using Bio Aerosol Mass Spectrometry (BAMS). Paper presented at the 2004 American Society of Microbiology Biodefense Meeting, Baltimore, MD.

36. Rasko, D. A., Ravel, J., Okstad, O. A., Helgason, E., Cer, R. Z., Jiang, L., et al. (2004). The genome sequence of Bacillus cereus ATCC 10987 reveals metabolic adaptations and a large plasmid related to Bacillus anthracis pXO1. Nucleic Acids Res, 32(3), 977-988.

37. Read, T. D., Peterson, S. N., Tourasse, N., Baillie, L. W., Paulsen, I. T., Nelson, K. E., et al. (2003). The genome sequence of Bacillus anthracis Ames and comparison to closely related bacteria. Nature, 423(6935), 81-86. 
38. Rosenbloom, M., Leikin, J. B., Vogel, S. N., \& Chaudry, Z. A. (2002). Biological and chemical agents: a brief synopsis. Am J Ther, 9(1), 5-14.

39. Rowe, C. A., Tender, L. M., Feldstein, M. J., Golden, J. P., Scruggs, S. B., MacCraith, B. D., et al. (1999). Array biosensor for simultaneous identification of bacterial, viral, and protein analytes. Anal Chem, 71(17), 3846-3852.

40. Samarawickrama, D. Y. (2002). Saliva substitutes: how effective and safe are they? Oral Dis, 8(4), 177-179.

41. Sewell, D. L. (2003). Laboratory safety practices associated with potential agents of biocrime or bioterrorism. J Clin Microbiol, 41(7), 2801-2809.

42. Simoes, E. A. (1999). Respiratory syncytial virus infection. Lancet, 354(9181), 847-852.

43. Sonenshein AL, H. J., Losick R. (1993). Bacillus subtilis and Other Gram-Positive Bacteria: Biochemistry, Physiology, and Molecular Genetics. Washington, DC: American Society for Microbiology.

44. Steele, P. T., Tobias, H. J., Fergenson, D. P., Pitesky, M. E., Horn, J. M., Czerwieniec, G. A., et al. (2003). Laser power dependence of mass spectral signatures from individual bacterial spores in bioaerosol mass spectrometry. Anal Chem, 75(20), 5480-5487.

45. Streckfus, C. F., \& Bigler, L. R. (2002). Saliva as a diagnostic fluid. Oral Dis, 8(2), 69-76.

46. Ullom, J. N., Frank, M., Gard, E. E., Horn, J. M., Labov, S. E., Langry, K., et al. (2001). Discrimination between bacterial spore types using time-of-flight mass spectrometry and matrix-free infrared laser desorption and ionization. Anal Chem, 73(10), 2331-2337.

47. Wilson, W. J., Strout, C. L., DeSantis, T. Z., Stilwell, J. L., Carrano, A. V., \& Andersen, G. L. (2002). Sequence-specific identification of 18 pathogenic microorganisms using microarray technology. Mol Cell Probes, 16(2), 119-127.

48. World Health Organization. (2002). World Health Report, 2002: Annex 2-Deaths by cause, sex and mortality stratum in WHO Regions, estimates for 2001; Annex 3-Burden of disease in DALYs by cause, sex and mortality stratum in WHO Regions, estimates for 2001. Retrieved 3/1/04, from http://www.who.int/whr/2002/annex/en/

49. World Health Organization. (2004). Tuberculosis: Disease Burden. Retrieved 3/1/05, from http://www.who.int/vaccine_research/diseases/tb/en/ 


\section{List of Figures}
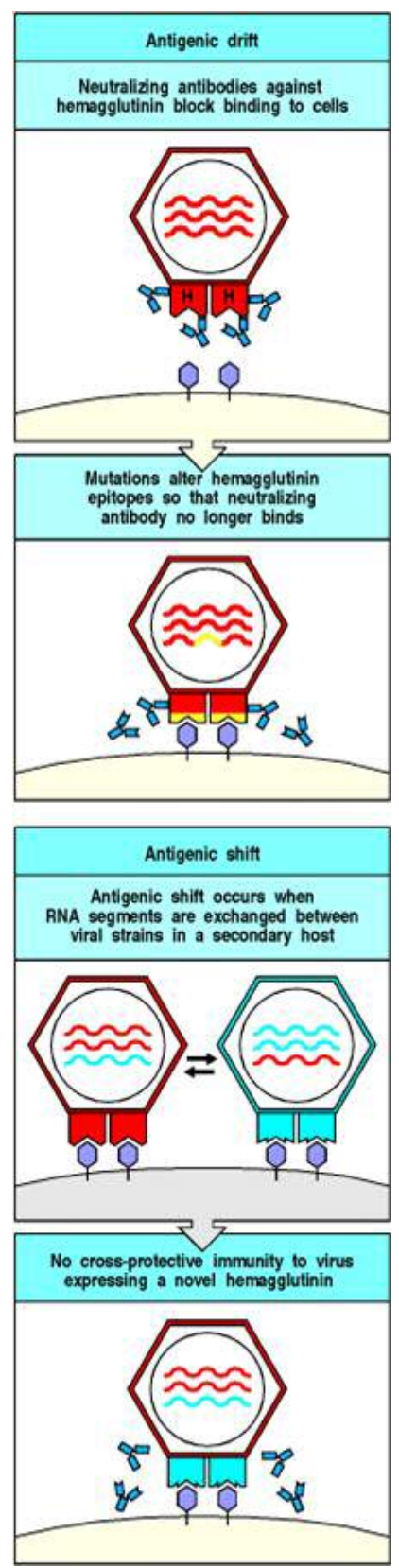

Figure 1. Influenza drift (above) and shift (below): from Immunobiology, 5th Edition 


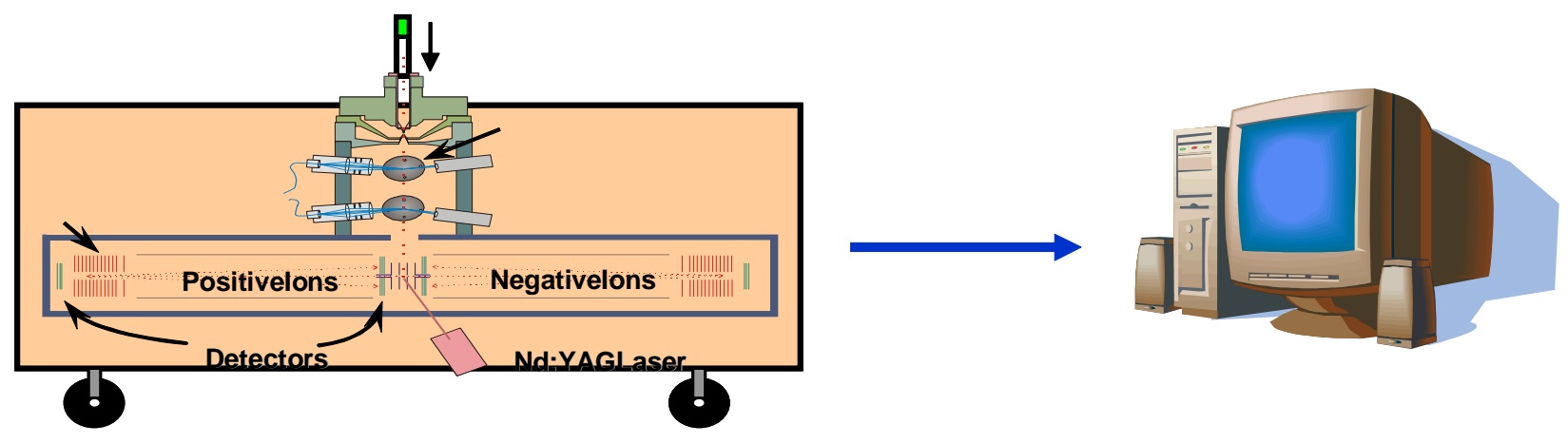

Figure 2. Schematic of the BAMS instrument in its current configuration.

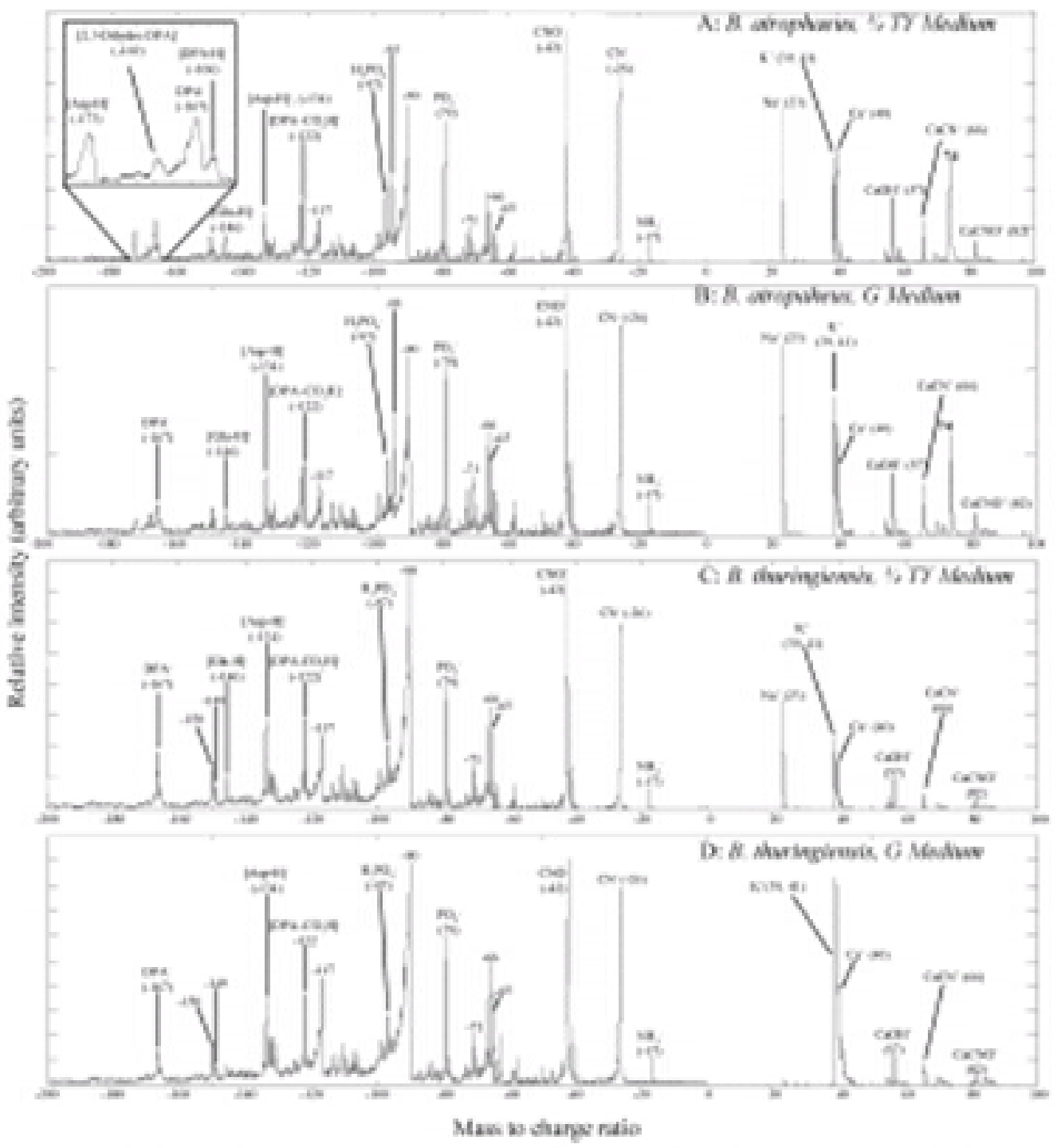

Figure 3. Positive and negative polarity average mass spectra of $B$. atrophaeus(A, B, 1000 spectra each) grown in 1/4 TY growth medium 
Equal, Growth Medum, Gelatin, Gold Band
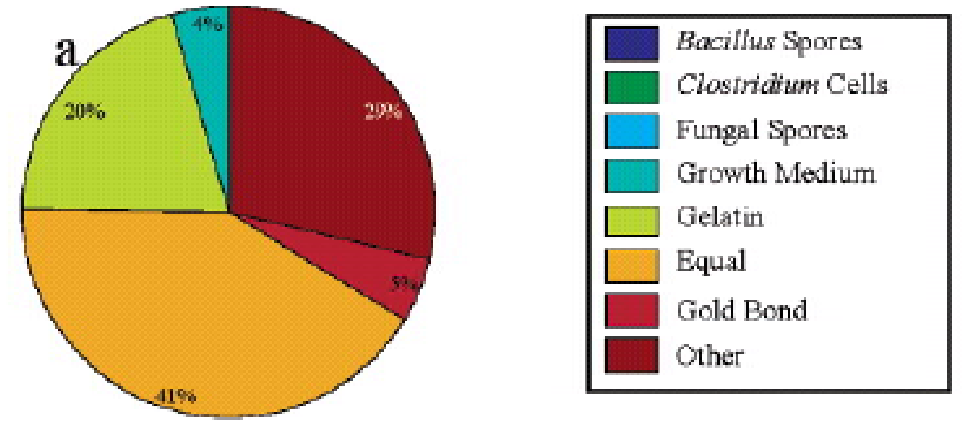

Bacillws, Baking Sodb and Fondered Sugat Added

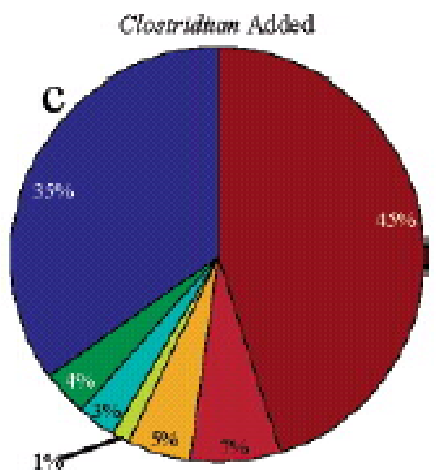

Figure 4. Pie charts showing real-time classifications of mixed aerosols. It is worth noting that fungal spores were not present in 

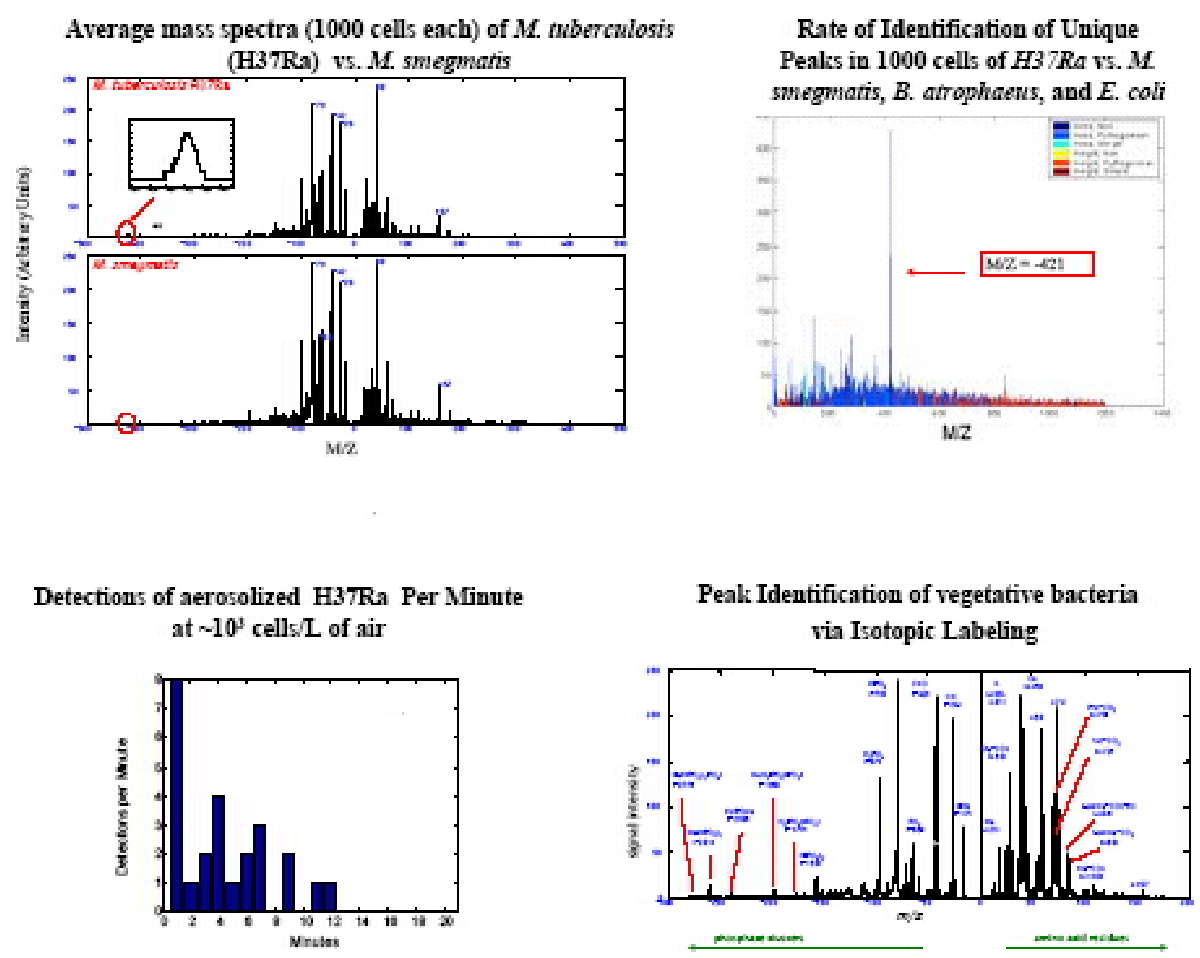

Figure 5. Preliminary work with Tuberculosis detection. From Pitesky et al, 2004 